\title{
MicroRNA profiling the resurrection plant Haberlea rhodopensis unveils essential regulators of survival under severe drought
}

\author{
E. APOSTOLOVA ${ }^{1}$, M. GOZMANOVA ${ }^{1}$, L. NACHEVA ${ }^{4}$, Z. IVANOVA ${ }^{2}$, V. TONEVA ${ }^{1,2}$, I. MINKOV ${ }^{2,3}$, \\ V. BAEV ${ }^{1}$, and G. YAHUBYAN ${ }^{1 *}$
}

Department of Plant Physiology and Molecular Biology, University of Plovdiv, BG-4000 Plovdiv, Bulgaria ${ }^{1}$ Institute of Molecular Biology and Biotechnologies, BG-4108 Markovo, Bulgaria ${ }^{2}$

Center of Plant Systems Biology and Biotechnology, BG-4000 Plovdiv, Bulgaria ${ }^{3}$

Fruit Growing Institute - Plovdiv, BG-4000 Plovdiv, Bulgaria ${ }^{4}$

\begin{abstract}
Small RNAs (sRNAs) are essential components of gene-regulatory networks, which guide plant development and tune it to environmental challenges. Though the past years have witnessed evidences on sRNA importance for stress response, there is scarce data on their involvement in resurrection plant survival under severe drought. Haberlea rhodopensis (hrh) is an angiosperm resurrection species, whose vegetative tissues can tolerate desiccation and recover upon rehydration. In this study, high-throughput sequencing sRNAs indicated a higher complexity of the sRNA population, especially of a 24 nt sRNA category, in the desiccated vegetative tissue of $H$. rhodopensis compared to unstressed tissues. The cross-species discovery was performed to predict 77 mature microRNAs (miRNAs), most of which were assigned to 23 high-confidence conserved miRNA families in the leaf tissue. Several members of the miR156/157, miR166, and miR399 families were found to be desiccation-responsive. The miR156/157 family members were found up-regulated upon dehydration and down-regulated upon rehydration, while the miR166 and miR399 family members followed an opposite trend of expression. A probable miR156/157 target, orthologous to the SQUAMOSA promoter binding proteinlike, was reconstructed in $H$. rhodopensis based on genomic data available for this species and the closely related Boea hygrometrica. Reverse transcription quantittative PCR analysis confirmed the expression profile of hrh-miR156a-5p and hrh-miR157-5p established by sRNA sequencing and revealed an inverse expression pattern between these miRNAs and their targets in the desiccated tissue. Our study suggests that the miR156/157 and miR399 families are essential for plant survival under severe drought due to their ability to control plant development and growth by modulating transcription factor expression.
\end{abstract}

Additional key words: desiccation tolerance, small RNAs, regulatory network.

\section{Introduction}

Drought has become one of the most important limiting factors to crop growth and productivity. After prolonged severe drought conditions, plants often fail to recover. Resurrection plants, a unique group of flora, have the extraordinary ability to survive extreme water shortages for years (Alpert 2000, Farrant 2007, Ryan 2017). Continuous research efforts have been made for understanding the mechanisms underlying desiccation tolerance in vegetative tissue of angiosperm resurrection plants (Scott 2000, Vicré et al. 2004, Farrant and Moore 2011, Gaff and Oliver 2013). The ultimate goal of these efforts is to speed up the development of crops with improved tolerance to drought stress. One of the most powerful approaches for crop improvement relies on the phenomenon of RNA interference involving sequence-specific gene regulation by small non-coding RNAs (Kamthan et al. 2015).

Small non-coding RNAs are RNA molecules of length less than 200 nucleotides (nt) that do not contain a peptideencoding open reading frame and therefore do not translate into proteins. However, these molecules are of paramount importance for genome functioning as they maintain genome integrity and control gene expression in the course of individual development and in response to changing conditions of the external and internal environment.

Submitted 22 January 2020, last revision 26 March 2020, accepted 21 April 2020.

Abbreviations: cDNA - complimentary DNA; miRNA - microRNA; nt - nucleotide; ORF - open reading frame; RWC - relative water content; SLRT-qPCR - stem-loop reverse transcription quantitative polymerase chain reaction; SPL - SQUAMOSA promoter binding protein-like; sRNA - small RNA; UTR - untranslated region; WGC - whole-genome shotgun contigs.

Acknowledgments: This project has received funding from the European Union's Horizon 2020 research and innovation programme under grant agreement No 739582 (Project PlantaSYST).

* Corresponding author; fax: (+359) 32261 560, e-mail: gyahubyan@uni-plovdiv.bg 
MicroRNAs (miRNAs) are a class of small RNAs comprising single-stranded molecules of $21-22 \mathrm{nt}$. In plants, hundreds of different miRNAs have been identified and grouped into families that are classified according to their conservation and diversification during evolution in the plant kingdom to two categories - evolutionally conserved miRNAs that are common to most plant species and non-conserved miRNAs existing in limited species (Axtell and Bowman 2008, Cuperus et al. 2011, Taylor et al. 2014, 2017).

Plant miRNA shave the potential for fine-tuning gene expression and transcriptome profileby either of two posttranscriptional mechanisms - mRNA decay or translational repression (Llave et al. 2002, Rhoades et al. 2002, Brodersen et al. 2008). Once in the cytoplasm, the miRNAs encounter mRNAs causing different shades of gene silencing and extending the control of the cell structure and function (Bartel 2018). Experimental studies in model and crop plant species have shown that miRNAs play a crucial role in vital biological processes being expressed in great diversity during development and growth, as well as in response to different abiotic and biotic stresses (Shukla et al. 2008, Rubio-Somoza and Weigel 2011, Khraiwesh et al. 2012). They are as well causal factors in evolution (Taylor et al. 2014), signal transduction (Navarro et al. 2006), and nutrient homeostasis (Chiou 2007).

Though past years have witnessed a lot of evidences on the essential role of miRNAs in abiotic stress response in various plant species (Phillips et al. 2007, Sunkar et al. 2007, Shriram et al. 2016), there is scarce data on the miRNA involvement in survival of resurrection plants under severe drought. The only data on the role of miRNAs in vegetative desiccation tolerance comes from the genome-wide investigation of miRNA transcriptome in the resurrection monocot Tripogon loliiformis (Njaci et al. 2018). In the present study, genome-wide identification of miRNAs was performed in Haberlea rhodopensis - a dicot resurrection species whose vegetative tissues can tolerate desiccation and recover upon rehydration. miRNA expression profiles were examined applying nextgeneration sequencing approach in hydrated, air-dried, and rehydrated vegetative tissues, and several differentially expressed miRNAs were validated by stem-loop reverse transcription quantitative PCR (SLRT-qPCR). Putative miRNA targets were predicted by seed region matching on transcripts of the closely related resurrection species $B$. hygrometrica. Further, drought-induced expression changes of one of the most reliable predicted target mRNAs were determined and correlated with corresponding miRNA transcription.

\section{Materials and methods}

Plants and treatments: Plants of Haberlea rhodopensis (Frv.) were propagated in vitro as previously described (Daskalova et al. 2011). Four-month old in vitro grown seedlings were transferred on acid soil and grown at a 16-h photoperiod, an irradiance of $100 \mu \mathrm{mol} \mathrm{m} \mathrm{m}^{-2} \mathrm{~s}^{-1}$, a temperature of $22^{\circ} \mathrm{C}$, and a relative humidity of $60 \%$ for two months. Drought treatment was started by stopping watering for $10 \mathrm{~d}$ until leaves looks air dry. Then the pots of desiccated plants were re-watered and covered for $24 \mathrm{~h}$. The percentage of relative water content (RWC) of leaf tissue was determined according Turner (1981). Leaf samples were collected at three-time points corresponding to the fully hydrated state $(\mathrm{H}$, about $90 \% \mathrm{RWC})$, the desiccated state (D, about $8 \%$ RWC) and the rehydrated state (R, about $75 \%$ RWC) of leaves. Three separate plants were used for each sampling point. The samples were frozen in liquid nitrogen and stored at $-80{ }^{\circ} \mathrm{C}$.

Extraction of RNA and high-throughput sequencing: Total RNA was extracted from leaf tissue with QIAzol lysis reagent (Qiagen, Hilden, Germany), followed by purification through a NucleoSpin miRNA column (Macherey-Nagel, Düren, Germany) according to the manufacturer's instructions. The RNA quality and quantity were checked on an Epoch microplate spectrophotometer (Bad Friedrichshall, Germany) and agarose gel. Three cDNA libraries were constructed and sequenced commercially on HiSeq2500 Illumina with TruSeq Small RNA library Prep (Macrogen, Seoul, South Korea).The small RNA data has been submitted in Sequence Read Archive (SRA, https://www.ncbi.nlm.nih.gov/sra) with BioProject accession number PRJNA548243.

Quality assessment of deep sequencing data and small RNA sequence analysis: Quality assessment of the sequencing libraries was done using FastQC (https:// www.bioinformatics.babraham.ac.uk/projects/fastqc/). Raw reads of the three datasets were adapter-cleaned using the FASTx toolkit (http://hannonlab.cshl.edu/fastx toolkit/) and low-quality reads were removed. Reads were counted and appended to each unique read following the conversion into FASTA format using FASTx toolkit. Only small RNA (sRNA) sequences of 15 to $30 \mathrm{nt}$ were retained for further analysis. For the identification of miRNAs, miRbase (Griffiths-Jones 2004) was used as a reference database. Copy numbers for the identified miRNAs were used to create gene table files feeding DESeq module for differential expression analysis (Anders and Huber 2010). The list of differentially expressed miRNAs was produced via DESeq by setting the threshold at $\log 2$ (fold change) $\leq-1.0$ or $\geq 1.0$ and $P$-value $\leq 0.05$ and miRNAs with less than 10 mean read number were filtered out. FASTQ/FASTA manipulations and differential expression analysis were carried out in the Galaxy platform (Afgan et al. 2018).

Bioinformatics analysis - databases and software: SQUAMOSA promoter binding protein-like (SPL) protein and mRNA sequences for $A$. thaliana, SPL putative protein sequences and whole-genome shotgun contigs (WGC) for $B$. hygrometrica were retrieved from the NCBI GeneBank (National Library of Medicine, NIH, Washington DC, USA). TBLASTN and BLASTP were used for comparing a protein query sequence to translated nucleotide sequence databases and protein sequence databases, respectively. For prediction of $H$. rhodopensis SPL-like mRNA sequence, short reads from $H$. rhodopensis nuclear 
genome sequencing (unpublished data) were mapped to the putative SPL-like mRNA sequence of B. hygrometrica using $B W A$ aligner with default options (Li and Durbin 2009). Mapped alignments were stored in the $B A M$ file and subsequently sorted. Next, unmapped reads were removed, and only mapped reads were obtained using SamTools $(\mathrm{Li}$ et al. 2009). The miRNA target prediction was achieved using the psRNATarget program (http://plantgrn.noble. org/psRNATarget/) with default parameters (Dai and Zhao 2011, Dai et al. 2018).

Expression analysis of miRNAs and predicted target genes using reverse transcription quantitative PCR: $1 \mu \mathrm{g}$ of total RNA was subjected to cDNA synthesis using Script cDNA polymerase (Bioscience, Jena, Germany and miRNA specific stem-loop primers designed according to Varkonyi-Gasic et al. (2007). The mixture was incubated at $16{ }^{\circ} \mathrm{C}$ for $30 \mathrm{~min}$ followed by 60 cycles: $30{ }^{\circ} \mathrm{C}$ for $30 \mathrm{~min}$, $42^{\circ} \mathrm{C}$ for $30 \mathrm{~s}, 50^{\circ} \mathrm{C}$ for $1 \mathrm{~s}$. The enzyme was inactivated at $70{ }^{\circ} \mathrm{C}$ for $5 \mathrm{~min}$. For subsequent expression analysis cDNA was diluted 10 times with nuclease-free water. The product was used as a template for qPCR performed on an Applied Biosystem 7500 system (Foster City, CA, USA) using a Green Master Mix kit (Genaxxon, Bioscience), miRNA-specific forward primer and universal reverse primer (Varkonyi-Gasic et al. 2007). The reaction mixtures were initially denatured at $95^{\circ} \mathrm{C}$ for $10 \mathrm{~min}$, followed by 40 cycles of $95^{\circ} \mathrm{C}$ for $15 \mathrm{~s}, 60^{\circ} \mathrm{C}$ for $1 \mathrm{~min}$.

For cDNA synthesis, $1 \mu \mathrm{g}$ of RNA was reverse transcribed with Script cDNA polymerase (Bioscience) following the manufacturer's instructions. For subsequent expression analysis cDNA was diluted 10 times with nuclease-free water. The qPCR reactions were carried out in a total volume of $25 \mathrm{~mm}^{3}$ and contained $4 \mathrm{~mm}^{3}$ of diluted cDNA, $1.5 \mathrm{~mm}^{3}$ of primer mix at a final concentration of $0.6 \mathrm{mM}, 4.5 \mathrm{~mm}^{3}$ of nuclease-free water and $12.5 \mathrm{~mm}^{3}$ of SYBR Green mix with $R O X$. PCR conditions: $50{ }^{\circ} \mathrm{C}$ for $2 \mathrm{~min}, 95^{\circ} \mathrm{C}$ for $10 \mathrm{~min}, 40$ amplification cycles of $95^{\circ} \mathrm{C}$ for $15 \mathrm{~s}$ and $60^{\circ} \mathrm{C}$ for $1 \mathrm{~min}$.

All reactions were performed in three technical replicates including a sample without a reverse transcription step (-RT) for each sequence. All primers are presented in Table 1 Suppl. Melting curve analysis was carried out for validation of specific amplification. The small RNA U6 gene was used as an endogenous control for normalization, and the untreated sample served as a reference. $\mathrm{Ct}$ values were calculated using the 7500-software v.2.0.1 (ABI, Foster City, CA, USA). Relative quantitation of gene expression (RQ) was determined applying the 2[-delta delta C(T)] method (Livak and Schmittgen 2001).

\section{Results}

Three sRNA libraries from vegetative tissue (green leaf) of unstressed fully hydrated (H), desiccated (D), and rehydrated $(\mathrm{R})$ plants were sequenced, thereby generating raw reads in millions $(\mathrm{M}): 51.4 \mathrm{M}(\mathrm{H}), 38.1 \mathrm{M}(\mathrm{D})$ and $52.9 \mathrm{M}(\mathrm{R})$. High-quality reads in the range of $35-45 \mathrm{M}$ reads per library were produced after removal of adaptors, low-quality sequences and sequences smaller than $15 \mathrm{nt}$ and longer than $30 \mathrm{nt}$ (Fig. $1 A$ ). Prior to read mapping, the redundant reads were collapsed to final datasets of unique reads, with an average of $1-3$ million per library, and copy numbers were associated (Fig. 1C). The majority of the high-quality tags were in the range of $21-24 \mathrm{nt}$ across the three libraries that were consistent with previous reports. Interestingly, while the $\mathrm{H}$ and $\mathrm{R}$ libraries showed a strong bias towards the enrichment of the reads with $24 \mathrm{nt}$, the D library disclosed relatively even distribution of the 21, 22, and 24 nt classes. The three libraries differed significantly with respect to non-redundant fraction, calculated as a number of distinct uniquely mapping reads/ total number of reads (Fig. $1 B$ ). The fraction was highest in the D library (37\%) and lowest in the H library (17\%). The length distribution of unique reads indicated the dominance of the $24 \mathrm{nt}$ read length in the three libraries with the highest value for the D library (58 \%, Fig. 1C).

Since $H$. rhodopensis genome has not yet been sequenced and transcriptome data are scarce, homology search for conserved miRNAs was done against unique mature miRNAs from the miRBase-21database set for green plants (Viridiplantae). In order to define miRNAs and their families in vegetative tissue of $H$. rhodopensis, the unique sequences of the three libraries were assembled in a single set and collapsed. Analysis of the three sRNA data sets identified 136 miRNAs candidates (Table 2 Suppl.) of which 77 were annotated as genuine guide miRNAs, 28 as isomiRs resulting from hairpin processing variations, and 31 as passenger miRNAs (miRNA*). All identified mature sequences are distributed in 28 miRNA families (Table 1), among which the miR166 and miR156/157 families contain the most members (15 and 14) and the most abundant are the miR166 (1956089 average reads per library), miR159 (541107 average reads per library) and miR396 (129002 average reads per library) families.

Expression changes of miRNAs upon desiccation and rehydration of $H$. rhodopensis were analysed by comparing normalized expression values of miRNAs between $\mathrm{H}$ and $\mathrm{D}$ plants using the $\mathrm{H}$ plants as a control sample (for identification of desiccation-responsive miRNAs), and between D and R plants using the D plants as a control sample (for identification of rehydrationresponsive miRNAs). According to the DESeq parameters described in Materials and Methods, 25 miRNAs were found to be differentially expressed during a desiccation/ rehydration event (Table 2). Upon desiccation, 13 miRNAs were up-regulated and 12 miRNAs were down-regulated compared with the unstressed plants. Only 4 miRNAs showed altered expressions upon subsequent rehydration. There was a clear correlation between the expressions of several members of miR156/157 and miR399families and of miR8175, and plant stress response. Several members of miR156/157 and miR8175 were up-regulated during desiccation, and down-regulated during subsequent rehydration, while miR399 had an opposite expression pattern - it was down-regulated during desiccation and up-regulated during rehydration. Among desiccationresponsive miRNAs, only one member of the miR166 family was identified presented by its passenger strand. 


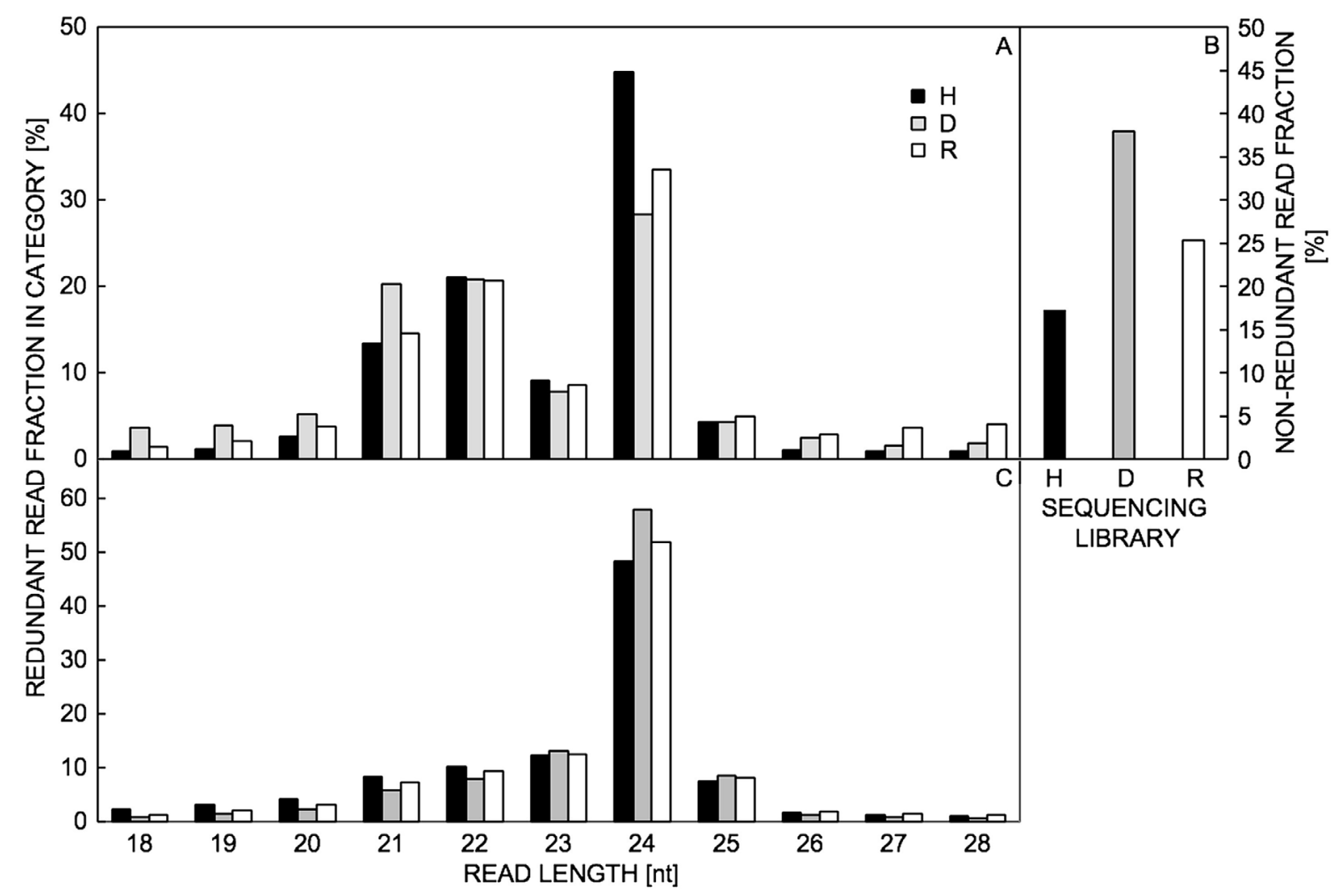

Fig. 1. Sequence length distribution in the Haberlea rhodopensis small RNA libraries. $A$ - The population of small RNAs separated in size classes given in the percentage of the total (redundant) read count; $B$ - relative frequencies of unique (non-redundant) reads given in the percentage of total read count; $C$ - population of small RNAs separated in size classes given in the percentage of unique read count. $\mathrm{H}$ - sequencing library of hydrated leaf tissue, D - sequencing library of desiccated leaf tissue, R - sequencing library of the leaf tissue rehydrated for $24 \mathrm{~h}$.

Table 1. Conserved microRNA (miRNA) families of Haberlea rhodopensis leaf tissue. High-confidence miRNAs are shown in bold.

\begin{tabular}{lc}
\hline miRNA family & Number of family members \\
\hline miR166 & 15 \\
miR156/157 & 14 \\
miR167, miR169 & 9 \\
miR160, miR396 & 7 \\
miR159, miR164, miR171, miR319, miR393 & 5 \\
miR168, miR390, miR399 & 4 \\
miR172, miR2111, miR397, miR408 & 2 \\
miR158, miR165, miR394, miR395, miR398, miR403, miR5139, miR6300, miR7972, miR8175 & 1 \\
\hline
\end{tabular}

Two members of the miR159 family were found to be affected by severe drought although their expressions were changed in opposite directions.

The expression patterns of several miRNAs were examined using SLRT-qPCR (Fig. 2). Among differentially expressed members of miR156/157 family in H. rhodopensis (hrh), hrh-miR156a-5p and hrh-miR157$5 \mathrm{p}$ were analyzed and found highly induced in response to desiccation and repressed upon rehydration(Fig. $2 A, B$ ) that was consistent with the expression profile established with small RNA-sequencing.
After validation of the differential expression of hrh-miR156a-5p and hrh-miR157-5p, the question that arose was whether it was possible to identify their likely target(s) in $H$. rhodopensis. The short SPL transcription factors - SPL3, SPL4, and SPL5 are some of the main targets of miR156/157 in A. thaliana and many other plant species (Preston and Hileman 2013). The difficulties of the miR156/157 target identification in $H$. rhodopensis were associated with the lack of genomic and transcriptomic data, but could still be overcome as we had raw data from the genome $H$. rhodopensis sequencing (unpublished data) 
Table 2. Differentially expressed microRNAs (miRNAs) in Haberlea rhodopensis leaf tissue during desiccation and rehydration. Upregulated miRNAs are shown on a grey background and down-regulated miRNAs on a white background. The miRNAs with log2 (fold change) $\leq-1.0$ or $\geq 1.0$ and $\mathrm{P}$-value $\leq 0.05$ were considered as significant.

\begin{tabular}{|c|c|c|c|c|}
\hline & miRNA & Sequence & Mean read number & $\begin{array}{l}\text { Fold change of } \\
\text { expression }(\log 2)\end{array}$ \\
\hline Desiccation & hrh-miR156a-5p & TTGACAGAAGATAGAGAGCAC & 30972.36 & 1.08 \\
\hline responsive & hrh-miR156b-5p & TGACAGAAGAGAGTGAGCACA & 131.67 & 1.17 \\
\hline \multirow[t]{23}{*}{ miRNAs } & hrh-miR156c-5p & TTGACAGAAGATAGATAGCAC & 26.04 & 1.16 \\
\hline & hrh-miR157-5p & TGACAGAAGATAGAGAGCAC & 254.41 & 1.6 \\
\hline & hrh-miR157-3p & GCTCTCTATGCTTCTGTCATC & 19.03 & 1.08 \\
\hline & hrh-miR159a-3p & CTTGGACTGAAGGGAGCTCCC & 82.12 & 1.3 \\
\hline & hrh-miR159b-3p & TTTGGATTGAAGGGAGCTCCT & 16.47 & -1.03 \\
\hline & hrh-miR160-3p & GCGTATGAGGAGCCAAGCATA & 56.87 & -2.24 \\
\hline & hrh-miR164-5p & TGGAGAAGCAGGGCACGTGCT & 54.9 & -1.55 \\
\hline & hrh-miR166-5p & GGAATGTTGTCTGGCTCGAGG & 92.81 & -1.98 \\
\hline & hrh-miR166-3p & TCGGACCAGGCTTCATTCCCT & 355.47 & -1.46 \\
\hline & hrh-miR167-5p & TGAAGCTGCCAGCATGATCTGA & 802.04 & -1.17 \\
\hline & hrh-miR168-5p & TCGCTTGGTGCAGGTCGGG & 84.16 & 1.77 \\
\hline & hrh-miR169-5p & CAGCCAAGGATGACTTGCC & 55.59 & 1.29 \\
\hline & hrh-miR171a-3p & TTGAGCCGCGCCAATATCACT & 382.5 & -1.26 \\
\hline & hrh-miR171b-3p & TGAGCCGAATCAATATCACTC & 20.54 & 1.36 \\
\hline & hrh-miR319-3p & CTTGGACTGAAGGGAGCTCCT & 709.55 & -1.31 \\
\hline & hrh-miR395-3p & CTGAAGTGTTTGGGGGAACTC & 184.28 & -1.09 \\
\hline & hrh-miR396-5p & TTCCACAGCTTTCTTGAACT & 878.03 & 1.15 \\
\hline & hrh-miR399a-3p & TGCCAAAGGAGAGTTGCCCTG & 2760.6 & -1.22 \\
\hline & hrh-miR399b-3p & TGCCAAAGGAGATTTGCCCCG & 774.02 & -2.02 \\
\hline & hrh-miR399c-3p & TGCCAAAGGAGATTTGCCCCT & 11.97 & -1.08 \\
\hline & hrh-miR5139-5p & AAACCTGGCTCTGATACCA & 22.54 & 1.17 \\
\hline & hrh-miR6300-3p & GTCGTTGTAGTATAGTGG & 47.1 & 1.73 \\
\hline & hrh-miR8175-3p & GATCCCCGGCAACGGCGCCA & 96.22 & 2.41 \\
\hline Rehydration & hrh-miR157-5p & TGACAGAAGATAGAGAGCAC & 260.96 & -1.06 \\
\hline responsive & hrh-miR160-3p & GCGTATGAGGAGCCAAGCATA & 28.15 & 1.08 \\
\hline \multirow[t]{2}{*}{ miRNAs } & hrh-miR399b-3p & TGCCAAAGGAGATTTGCCCCG & 474.06 & 1.26 \\
\hline & hrh-miR8175-3p & GATCCCCGGCAACGGCGCCA & 105.97 & -1.19 \\
\hline
\end{tabular}

and genomic and transcriptomic data from the closely related $B$. hygrometrica (Xiao et al. 2015).

First, TBLASTN was performed using A. thaliana SPL3/4/5protein sequences as query sequences against the whole-genome shotgun contigs (WGC) of B.hygrometrica. Among the sequences producing significant alignments, the region from 9552 - $10863 \mathrm{nt}$ of contig 20643 of $B$. hygrometrica, cv. XS01, showed the highest percentage of identity. The region was extracted in FASTA and open reading frames (ORFs) were used to determine protein-coding boundaries and reconstruct the putative $B$. hygrometrica mRNA (Fig. 1 Suppl.). BLASTP of the ORF of the putative mRNA against all predicted $B$. hygrometrica proteins showed $100 \%$ identity to the protein F511 21490, and the presence of the highly conserved SQUAMOSA promoter binding protein domain (Fig. 3B). When compared to $A$. thaliana SPL3/4/5, the putative $B$. hygrometrica protein showed the highest degree of sequence similarity with AthSPL3, and so it was named as SPL3-like.

Second, bearing in mind that the miR157/156 target sites are located in the 3'-UTRs of the SPL3/4/5 mRNAs of $A$. thaliana, the 3 '-end of $B$. hygrometrica mRNA was extended with $100 \mathrm{nt}$ by expanding the boundaries of the 


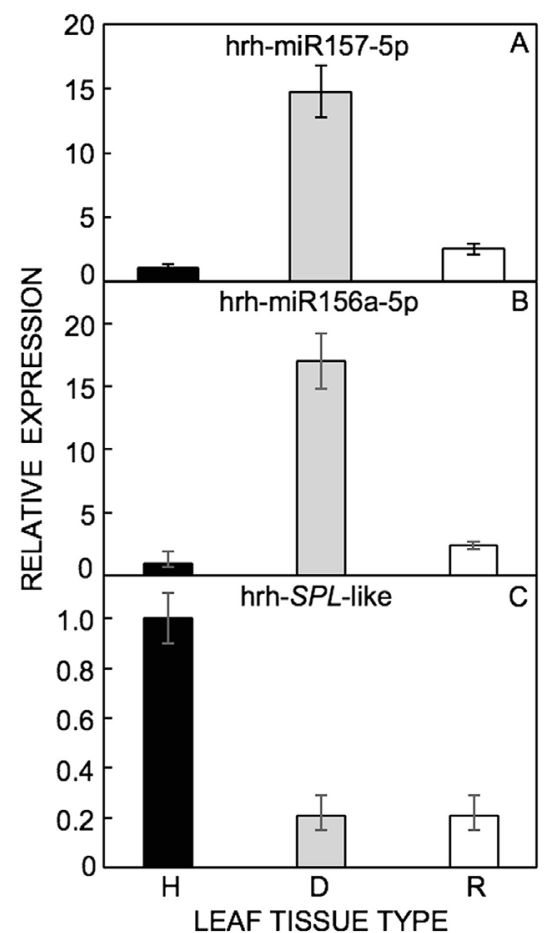

Fig. 2. Expression changes of miR156/157 family members and SQUAMOSA promoter binding protein (SPL) 3-like in Haberlea rhodopensis during desiccation and rehydration. Relative transcriptions of hrh-miR156a-5p $(A)$ and hrh-miR157-5p $(B)$ determined by stem-loop reverse transcription quantitative PCR, and SPL3-like mRNA $(C)$ determined by reverse transcription quantitative PCR. H - hydrated leaf tissue, D - desiccated leaf tissue, $\mathrm{R}$ - leaf tissue rehydrated for $24 \mathrm{~h}$. small nuclear RNA U6 was used as an internal control. Error bars represent standard deviation from three independent experiments.

region extracted from contig 20643. The putative SPL3like mRNA was searched by psRNATarget for target sites with all miRNAs of miRBase (release 21). The outcome indicated a miR156/157 binding site in the 3'-UTR with the highest expected score of 0.0 (Fig. $3 A$, Table 3 Suppl.).

In order to predict SPL3-like mRNA in H. rhodopensis, sequencing reads produced by the genome $H$. rhodopensis sequencing were mapped to B.hygrometrica SPL3-like mRNA. The partial SPL3-like mRNA of $H$. rhodopensis was reconstructed (Fig. 1 Suppl.) and confirmed by sequencing. The ORF indicated the SBP domain in the protein molecule. Alignment of the putative SPL3-like proteins of $B$. hygrometrica and $H$. rhodopensis revealed very high sequence identity (Fig. 3B,C). RT-qPCR analysis showed that the SPL3-like of $H$. rhodopensis was strongly repressed in response to desiccation and remained unchanged during the first $24 \mathrm{~h}$ of rehydration (Fig. 2C).

\section{Discussion}

Regulatory RNAs, including miRNAs, are seen as key intermediaties in the epigenetic network emerging as a crucial component of plant stress response and adaptation (Grant-Downton and Dickinson 2005). The identification of the entire repertoire of miRNAs in plant tissue, as well as the relative amount of each of them, is possible thanks to the high-throughput sequencing techniques. This has led to the accumulation of many data implying the role of miRNAs in plant response to adverse environmental conditions including drought (Ding et al. 2013, Ferdous et al. 2015). These studies outline the drought-responsive miRNAs, but since they have been conducted in species susceptible or tolerant to mild or moderate drought stress, and the question of the importance of these regulatory molecules for surviving of plants under extremely dry conditions needs to be further explored. So far, genomewide profiling of desiccation-responsive miRNAs has been conducted in the resurrection monocotyledonous plant T. loliiformis (Njaci et al. 2018) and numbers of desiccation-specific miRNAs have been detected. Since miRNA responsiveness to desiccation stress may be species-specific, for example as observed in drought stress (Ferdous et al. 2015), it is worth to expand miRNA analyses to other members of the group of resurrection plants. In this study, we describe the miRNA repertoire specific for vegetative tissue of the resurrection dicotyledonous plant $H$. rhodopensis and its expression changes under conditions of desiccation and subsequent rehydration.

Two major classes (21 - $22 \mathrm{nt}$ and $24 \mathrm{nt}$ ) of sRNAs were revealed in the three libraries that were typical for plants. The 21 - 22 nt sRNAs were highly redundant while the $24 \mathrm{nt}$ sRNAs were much less redundant. The portion of the unique sRNAs in the total read number was greatest in the D library compared to the other two libraries thus indicating increasing complexity of the sRNA population during desiccation of $H$. rhodopensis. Moreover, the portion of $24 \mathrm{nt}$ sRNAs in the unique read number was greater in the desiccated sample compared to the other two samples. The great majority of $24 \mathrm{nt}$ sRNAs are DCL3-processed cis-acting small-interfering RNAs (siRNAs) that derive from heterochromatin, transposons and repeat elements (Reinhart et al. 2002, Xie et al. 2004). Our result indicates that $24 \mathrm{nt}$ sRNAs (most likely si-RNAs) production from various loci is activated during desiccation, presumably to protect genome integrity by RNA-dependent DNAmethylation or transposon silencing (Pontier et al. 2005, Qi et al. 2006, Chen et al. 2009).

Due to the fact that a great number of miRNAs have orthologues in the plant kingdom, the cross-species discovery was performed to identify conserved miRNAs, and assign their families (Taylor et al. 2017), in leaf tissue of $H$. rhodopensis. Of all 27 identified families, 23 families fall within the group of eudicot miRNA families of miRBase 21 considered as highly confident (Axtell and Meyers 2018). The expressions of miRNA families were evaluated in $H$. rhodopensis leaf tissue based on the counts observed in the three libraries. Although the number of reads is dependent on the sequencing depth applied, comparative representation of each miRNA is not expected to be distorted. The most abundant miRNA family in H. rhodopensis green tissue was miR166 (approximately $2 \mathrm{M}$ reads/library), followed by miR159 (approximately $0.5 \mathrm{M}$ reads/library) and miR399 (apx. 0.15 M reads/ library). 


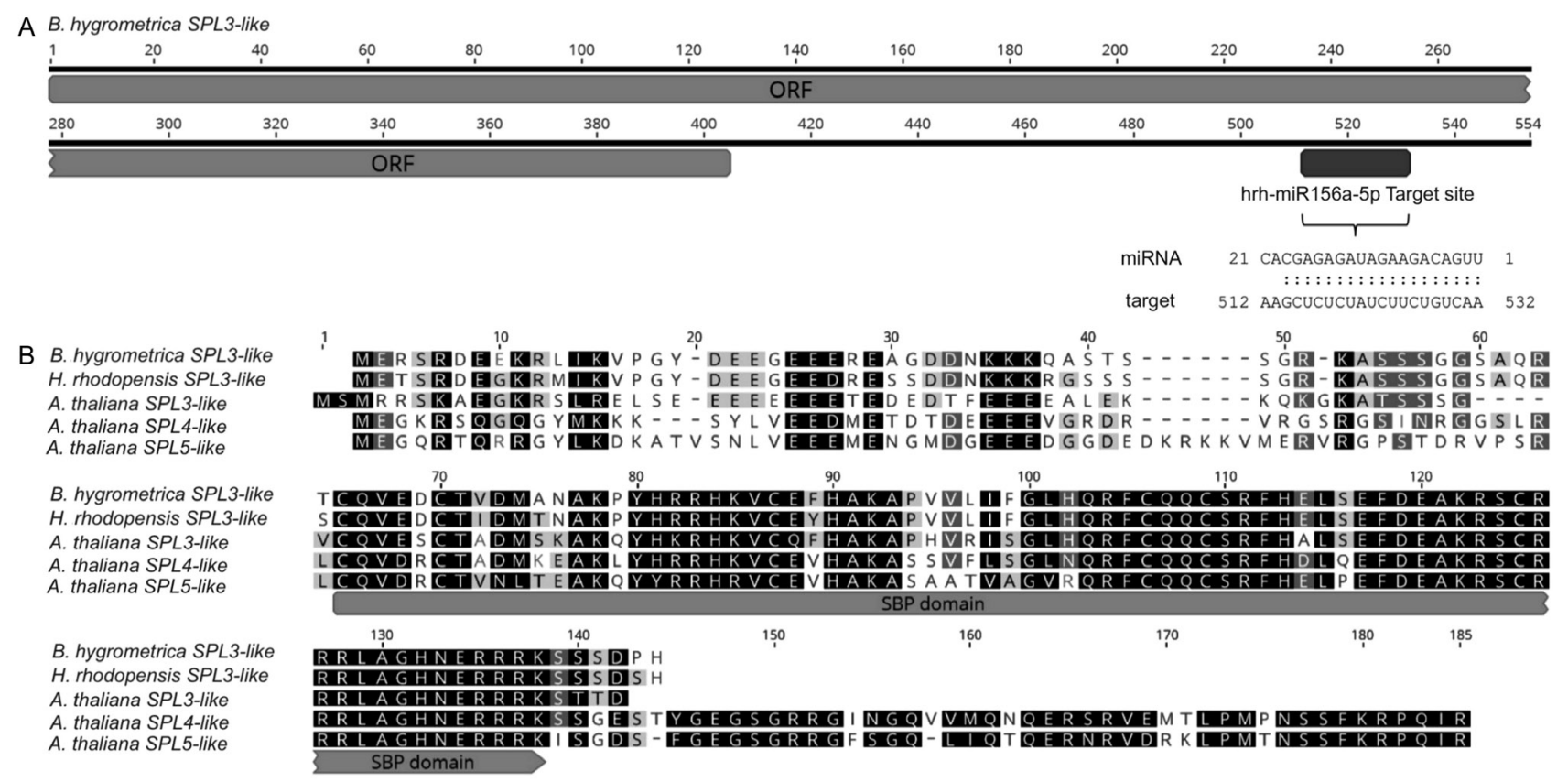

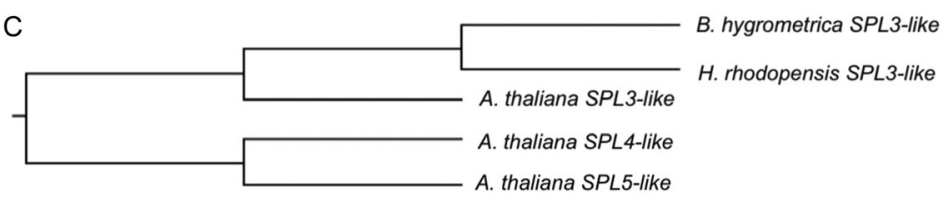

Fig. 3. The prediction of SPL3-like orthologs in Boea hygrometrica and Haberlea rhodopensis. A - The putative SPL3-like mRNA of $B$. hygrometrica, an open reading frame and an 156/157 binding site indicated; $B$ - The protein alignment of SPL3/4/5 paralogs of Arabidopsis thaliana and the predicted SPL3-like orthologs of B. hygrometrica and H. rhodopensis, the highly conserved SQUAMOSA promoter binding protein (SPB) domain indicated; $C$ - the phylogenetic tree of SQUAMOSA promoter binding protein-like (SPL) proteins analyzed in the three species.

OPTIMAL CONDITIONS

miR156/157 miR399

low expressions high levels

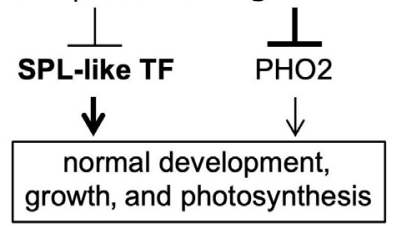

Fig. 4. The role of miR156/157 and miR399 in Haberlea rhodopensis desiccation response. Under normal conditions, low expressions of miR156/157 have no effect on SQUAMOSA promoter binding protein-like (SPL) accumulation necessary for normal growth and development whereas up-regulated miR399 represses phosphate 2 (PHO2) in order to serve to phosphate uptake and allocation, and photosynthetic activity. During severe drought stress, up-regulated miR156/157 represses the SPL gene expression to attenuate plant growth and development whereas down-regulated miR399 de-represses $\mathrm{PHO} 2$ synthesis resulting in decreased phosphate uptake and photosynthetic activity.

We identified 136 miRNAcandidates in H. rhodopensis, previously 196 miRNAs were predicted in the closely related $B$. hygrometrica (Xiao et al. 2015), but the question of which particular miRNA could be of physiological consequence for the plant in response to severe drought was unclear. From our data, several miRNA families appear to contribute for desiccation tolerance and plant survival under severe water deficit. The miR399 family targets the gene phosphate 2, which codes for ubiquitin-conjugating E2 enzyme and regulates phosphate homeostasis (Chiou et al. 2006, Pant et al. 2008). Over expression of miR399 affects phosphate acquisition by roots, its allocation from roots to shoots and impairs its remobilization out of old leaves (Chiou et al. 2006). In our study, several members of miR399 family were down-regulated in the desiccated tissue of $H$. rhodopensis and up-regulated upon re-hydration. We can speculate that repression of miR399 may counteract phosphate uptake and phosphate allocation from root to leaf in $H$. rhodopensis under severe drought stress and thus reduce photosynthesis and photooxidative damage in desiccated leaves. Coupling decreased photosynthetic activity with physiological and metabolic changes is one of the main strategies of resurrection plants for protection of the photosynthetic machinery upon desiccation (Farrant et al. 2015, Challabathula et al. 2016, Georgieva et al. 2007). The observed induction of miR399 during subsequent re-watering of $H$. rhodopensis could de-repress phosphate uptake and its allocation to leaves stimulating photosynthesis and growth. 
It was shown that miR159-MYB33/65 pathway was ubiquitously present throughout rosette development of Arabidopsis without having a clear functional role in leaf development or general stress response (Li et al. 2016). Since our RNAseq data indicated opposite changes of gene expression of two miR159 members in the airdried tissue, it appears that miR159 family does not play a role in $H$. rhodopensis desiccation response. Yet there are studies reporting expression changes of miR159 in response to drought in potato (Yang et al. 2014) and maize (Wang et al. 2014). So, we cannot rule out that miR159MYB33/65 pathway may still take part in the early stages of dehydration of resurrection plants when leaves shrink and curl up.

In plants, miR156 and miR157 have been grouped in one miRNA family because of their high degree of sequence similarity and their conserved target - SPL family. The family members are transcription factors that play an essential role in plant growth and development (Preston and Hileman 2013). The optimal time for developmental and morphological adaptive changes can be determined by certain environmental cues affecting the regulatory circuit around the miR156/157-SPL module (Wang and Wang 2015). Expression of several miR156/157 family members and SPL factors has been reported to be regulated by various stresses factors. Drought-responsiveness of the miR156/SPL circuit has been shown in barley (Kantar et al. 2010), alfalfa (Arshad et al. 2017), and mulberry (Li et al. 2017). Recently, some members of the mi156/157 and SPL families were predicted to have desiccation tolerancerelated functions in the resurrection plant $T$. loliiformis (Njaci et al. 2018). In our study, RNAseq data validated by RT-qPCR demonstrated desiccation-dependent induction of several members of the mi156/157 family and their repression by subsequent rehydration in $H$. rhodopensis.

Increased miR156/157 activity resulted in downregulation of the predicted SPL3 orthologue in $H$. rhodopensis desiccated tissue. In the first $24 \mathrm{~h}$ of rehydration when miRNA content fell, the SPL expression remains low as it was most likely under the control of regulatory factors that did not yet responded to changing environmental conditions. Our observations extended the idea of Wang and Wang (2015), which treats the miR156/157-SPL circuit as a regulatory hub linking various endogenous developmental cues and external signals to control plant developmental processes, in the case of resurrection plants to achieve vegetative desiccation tolerance and recover under favourable conditions.

\section{Conclusions}

In $H$. rhodopensis, severe drought induces transcriptome reprogramming with functional and structural consequences associated with photosynthetic activity, energy metabolism, primary and secondary metabolic processes, plant hormone signal transduction, proteinquality control and DNA repair (Gechev et al. 2013, Liu et al. 2018). Here, we provide evidences that miRNAs can enhance reprogramming efficiency via post- transcriptional regulation of desiccation-responsive genes. The miR156/157 and miR399 families are essential for plant survival under severe water stress due to their ability to control plant development and growth by modulating transcription factor expression (Fig. 4). It is important to precisely characterize the miR $156 / 157-$ SPL regulatory circuit in the resurrection plants, for example to define the SPL orthologous and paralogous genes, to answer if there is a link between the miRNA-mediated SPL response and sugar accumulation by testing leaves of different age or between the early and late plant responses to water deprivation (Yang et al. 2013), between anthocyanin synthesis and miR156/157-targeted SPL transcription factors (Gou et al. 2011), etc. Desiccation-responsive regulatory modules of miRNAs and cognate transcription factors seem to be promising targets of genetic manipulation for improving plant performances under drought conditions.

\section{References}

Afgan, E., Baker, D., Batut, B., Van den Beek, M., Bouvier, D., Cech, M., Chilton, J., Clements, D., Coraor, N., Grüning,B.A., Guerler, A., Hillman-Jackson, J., Hiltemann,S., Jalili,V., Rasche, H., Soranzo, N., Goecks, J., Taylor, J., Nekrutenko, A., Blankenberg, D.: The Galaxy platform for accessible, reproducible and collaborative biomedical analyses. - Nucl. Acids Res. 46(Suppl.): W537-W544, 2018.

Alpert, P.: The discovery, scope, and puzzle of desiccation tolerance in plants. - Plant Ecol. 151: 5-17, 2000.

Anders, S., Huber, W.: Differential expression analysis for sequence count data. - Genome Biol. 11 (Suppl.): R106, 2010.

Arshad, M., Feyissa, B.A., Amyot, L., Aung, B., Hannoufa, A.: MicroRNA156 improves drought stress tolerance in alfalfa (Medicago sativa) by silencing SPL13. - Plant Sci. 258: 122136, 2017.

Axtell, M.J., Meyers, B.C.: Revisiting criteria for plant microRNA annotation in the era of big data. - Plant Cell 30: 272-284, 2018.

Axtell, M.J., Bowman, J.L.: Evolution of plant microRNAs and their targets. - Trends Plant Sci. 13: 343-349, 2008.

Bartel, D.P.: Metazoan MicroRNAs. - Cell 173: 20-51, 2018.

Brodersen, P., Sakvarelidze-Achard, L., Bruun-Rasmussen, M., Dunoyer, P., Yamamoto, Y.Y., Sieburth, L., Voinnet, O.: Widespread translational inhibition by plant miRNAs and siRNAs. - Science 320: 1185-1190, 2008.

Challabathula, D., Puthur, J.T., Bartels, D.: Surviving metabolic arrest: photosynthesis during desiccation and rehydration in resurrection plants. - Ann. N. Y. Acad. Sci. 1365: 89-99, 2016.

Chen, C., Jin, J., James, D.A., Adams-Cioaba, M.A., Park, J.G., Guo, Y., Tenaglia, E., Xu, C., Gish, G., Min, J., Pawson, T.: Mouse Piwi interactome identifies binding mechanism of Tdrkh Tudor domain to arginine methylated Miwi. - Proc. nat. Acad. Sci. USA 106: 20336-20341, 2009.

Chiou, T.: The role of microRNAs in sensing nutrient stress. Plant Cell Environ. 30: 323-332, 2007.

Chiou, T.J., Aung, K., Lin, S.I., Wu, C.C., Chiang, S.F., Su, C.L.: Regulation of phosphate homeostasis by microRNA in Arabidopsis. - Plant Cell 18: 412-421, 2006.

Cuperus, J.T., Fahlgren, N., Carrington, J.C.: Evolution and functional diversification of MIRNA genes. - Cell 23: 431442, 2011.

Dai, X., Zhao, P.X.: psRNAtarget: a plant small RNA target analysis server. - Nucl. Acids Res. 39: W155 - W159, 2011. 
Dai, X., Zhuang, Z., Zhao, P.X.: psRNAtarget: a plant small RNA target analysis server (2017 release). - Nucl. Acids Res. 46: W49-W54, 2018.

Daskalova, E., Dontcheva, S., Yahoubyan, G., Minkov, I., Toneva, V.: A strategy for conservation and investigation of the protected resurrection plant Haberlea rhodopensis.Friv. BioRisk 6: 41-60, 2011.

Ding, Y., Tao, Y., Zhu, C.: Emerging roles of microRNAs in the mediation of drought stress response in plants. - J. exp. Bot. 64: 3077-3086, 2013.

Farrant, J.M.: Mechanisms of desiccation tolerance in angiosperm resurrection plants. - In: Jenks, M.A., Wood, A.J. (ed.): Plant Desiccation Tolerance. Pp. 51 - 90. Blackwell Publishing, Ames 2007.

Farrant, J.M., Cooper, K., Hilgart, A., Abdalla, K.O., Bentley, J., Thomson, J.A., Dace, H.J., Peton, N., Mundree, S.G., Rafudeen, M.S.: A molecular physiological review of vegetative desiccation tolerance in the resurrection plant Xerophyta viscosa (Baker). - Planta 42: 407-426, 2015.

Farrant, J.M., Moore, J.P.: Programming desiccation-tolerance: from plants to seeds to resurrection plants. - Curr. Opin. Plant Biol. 14: 340-345, 2011.

Ferdous, J., Hussain, S.S., Shi, B.: Role of microRNAs in plant drought tolerance. - Plant biotechnol. J. 13: 293-305, 2015.

Gaff, D.F., Oliver, M.J.: The evolution of desiccation tolerance in angiosperm plants: a rare yet common phenomenon. - Funct. Plant Biol. 40: 315-328, 2013.

Gechev, T.S., Benina, M., Obata, T., Tohge, T., Sujeeth, N., Minkov, I., Hille, J., Temanni, M.R., Marriott, A.S., Bergström, E.: Molecular mechanisms of desiccation tolerance in the resurrection glacial relic Haberlea rhodopensis. - Cell Mol. Life Sci. 70: 689-709, 2013.

Georgieva, K., Szigeti, Z., Sarvari, E., Gaspar, L., Maslenkova, L., Peeva, V., Peli, E., Tuba, Z.: Photosynthetic activity of homoiochlorophyllous desiccation tolerant plant Haberlea rhodopensis during dehydration and rehydration. - Planta 225: 955-964, 2007.

Gou, J.Y., Felippes, F.F., Liu, C.J., Weigel, D., Wang, J.W.: Negative regulation of anthocyanin biosynthesis in Arabidopsis by a miR156-targeted SPL transcription factor. Plant Cell 23: 1512-1522, 2011.

Grant-Downton, R.T., Dickinson, H.G.: Epigenetics and its implications for plant biology. 1. The epigenetic network in plants. - Ann. Bot. 96: 143-1164, 2005.

Griffiths-Jones, S.: The microRNA registry. - Nucl. Acids Res. 32(Suppl.): D109-D111, 2004.

Kamthan, A., Chaudhuri, A., Kamthan, M., Datta, A.: Small RNAs in plants: recent development and application for crop improvement. - Front. Plant Sci. 6: 208, 2015.

Kantar, M., Unver, T., Budak, H.: Regulation of barley miRNAs upon dehydration stress correlated with target gene expression. - Funct. integr. Genomics 10: 493, 2010.

Khraiwesh, B., Zhu, J.K., Zhu, J.: Role of miRNAs and siRNAs in biotic and abiotic stress responses of plants. - Biochim. biophys. Acta 1819: 137-148, 2012.

Li, H., Durbin, R.: Fast and accurate short read alignment with Burrows-Wheeler transform. - Bioinformatics 25: 1754-1760, 2009.

Li, H., Handsaker, B., Wysoker, A., Fennell, T., Ruan, J., Homer, N., Marth, G., Abecasis, G., Durbin, R.: 1000 genome project data processing subgroup: the sequence alignment/map format and SAM tools. - Bioinformatics 25: 2078-2079,2009.

Li, R., Chen, D., Wang, T., Wan, Y., Li, R., Fang, R., Zhao, W.: High throughput deep degradome sequencing reveals microRNAs and their targets in response to drought stress in mulberry (Morus alba). - PloS ONE 12: e0172883, 2017.
Li, Y., Alonso-Peral, M., Wong, G., Wang, M.B., Millar, A.A.: Ubiquitous miR159 repression of MYB33/65 in Arabidopsis rosettes is robust and is not perturbed by a wide range of stresses. - BMC Plant Biol. 16: 179, 2016.

Liu, J., Moyankova, D., Lin, C.T., Mladenov, P., Sun, R.Z., Djilianov, D., Deng, X.: Transcriptome reprogramming during severe dehydration contributes to physiological and metabolic changes in the resurrection plant Haberlea rhodopensis. BMC Plant Biol. 18: 351, 2018.

Livak, K.J., Schmittgen, T.D.: Analysis of relative gene expression data using real-time quantitative PCR and the 2(-delta delta C(T)) method. - Methods 25: 402-408, 2001.

Llave, C., Kasschau, K., Rector, M.A., Carrington, J.C.: Endogenous and silencing-associated small RNAs in plants. - Plant Cell 14: 1605-1619, 2002.

Navarro, L., Dunoyer, P., Jay, F., Arnold, B., Dharmasiri, N., Estelle, M., Voinnet, O., Jones, J.D.: A plant miRNA contributes to antibacterial resistance by repressing auxin signalling. - Science 312: 436-439, 2006.

Njaci, I., Williams, B., Castillo-González, C., Dickman, M.B., Zhang, X., Mundree, S.: Genome-wide investigation of the role of microRNAs in desiccation tolerance in the resurrection grass Tripogon loliiformis. - Plants 7: 68, 2018.

Pant, B.D., Buhtz, A., Kehr, J., Scheible, W.R.: MicroRNA399 is a long-distance signal for the regulation of plant phosphate homeostasis. - Plant J. cell. mol. Biol. 53: 731-738, 2008.

Phillips, J.R., Dalmay, T., Bartels, D.: The role of small RNAs in abiotic stress. - FEBS Lett. 581: 3592-3597, 2007.

Pontier, D., Yahubyan, G., Vega, D., Bulski, A., Saez-Vasquez, J., Hakimi, M.A., Lerbs-Mache, S., Colot, V., Lagrange, T.: Reinforcement of silencing at transposons and highly repeated sequences requires the concerted action of two distinct RNA polymerases IV in Arabidopsis. - Genes Dev. 19: 2030-2040, 2005.

Preston, J.C., Hileman, L.C.: Functional evolution in the plant Squamosa-promoter binding protein-like (SPL) gene family. - Front. Plant Sci. 4: 80, 2013.

Qi, Y., He, X., Wang, X.J., Kohany, O., Jurka, J., Hannon, G.J.: Distinct catalytic and non-catalytic roles of ARGONAUTE4 in RNA-directed DNA methylation. - Nature 443: 1008-1012, 2006.

Reinhart, B.J., Weinstein, E.G., Rhoades, M.W., Bartel, B., Bartel, D.P.: MicroRNAs in plants. - Genes Dev. 16: 16161626, 2002.

Rhoades, M., Reinhart, B., Lim, L., Burge, C., Bartel, B., Bartel, D.: Prediction of plant microRNA targets. - Cell 110: 513$520,2002$.

Rubio-Somoza, I., Weigel, D.: MicroRNA networks and developmental plasticity in plants. - Trends Plant Sci. 16: 258-64, 2011.

Ryan, J.C.: A comparative history of resurrection plants. CLCWeb: Comparative Literature and Culture 19: 1, 2017.

Scott, P.: Resurrection plants and the secrets of eternal leaf. Ann. Bot. 85: 159-166, 2000.

Shriram, V., Kumar, V., Devarumath, R.M., Khare, T.S., Wani, S.H.: MicroRNAs as potential targets for abiotic stress tolerance in plants. - Front. Plant Sci. 7: 817, 2016.

Shukla, L.I., Chinnusamy, V., Sunkar, R.: The role of microRNAs and other endogenous small RNAs in plant stress responses. -Biochim. biophys. Acta 1779: 743-748, 2008.

Sunkar, R., Chinnusam, V., Zhu, J., Zhu, J.K.: Small RNAs as big players in plant abiotic stress responses and nutrient deprivation. - Trends Plant Sci. 12: 301-309, 2007.

Taylor, R.S., Tarver, J.E., Hiscock, S.J., Donoghue, P.C.: Evolutionary history of plant microRNAs. - Trends Plant Sci. 19: 175-182, 2014. 
Taylor, R.S., Tarver, J.E., Foroozani, A., Donoghue, P.C.J.: MicroRNA annotation of plant genomes - Do it right or not at all. - BioEssays 39: 1600113, 2017.

Turner, N.C.: Techniques and experimental approaches for the measurement of plant water stress. - Plant Soil 58: 339-366, 1981.

Varkonyi-Gasic, E., Wu, R., Wood, M., Walton E.F., Hellens, R.P.: Protocol: a highly sensitive RT-PCR method for detection and quantification of microRNAs. - Plant Methods 3: 12, 2007.

Vicré, M., Lerouxel, O., Farrant, J.M., Lerouge, P., Driouich, A.: Composition and desiccation-induced alterations of the cell wall in the resurrection plant Craterostigma wilmsii. Physiol. Plant. 120: 229-239, 2004

Wang, H., Wang, H.: Molecular plant miR156/SPL module, a regulatory hub and versatiletToolbox. Gears up crops for enhanced agronomic traits. - Mol. Plant 8: 677-688, 2015.

Wang, Y.G., An, M., Zhou, S.F., She, Y.H., Li, W.C., Fu, F.L.: Expression profile of maize microRNAs corresponding to their target genes under drought stress. - Biochem. Genet. 52:
474-93, 2014.

Xiao, L., Yang, G., Zhang, L., Yang, X., Zhao, S., Ji, Z., Zhou, Q., Hu, M., Wang, Y., Chen, M., Xu, Y., Jin, H., Xiao, X., Hu, G., Bao, F., Hu, Y., Wan, P., Li, L., Deng, X., Kuang, T., Xiang, C., Zhu, J.K., Oliver, M.J., He, Y.: The resurrection genome of Boea hygrometrica: a blueprint for survival of dehydration. - Proc. nat. Acad. Sci. USA 112: 5833-5837, 2015.

Xie, Z., Johansen, L.K., Gustafson, A.M., Kasschau, K.D., Lellis, A.D., Zilberman, D., Jacobsen, S.E., Carrington, J.C. Genetic and functional diversification of small RNA pathways in plants. - PloS Biol. 2: E104, 2004.

Yang, J., Zhang, N., Mi, X., Wu, L., Ma, R., Zhu, X., Yao, L., Jin, X., Si, H., Wang, D.: Identification of miR159s and their target genes and expression analysis under drought stress in potato. - Comput. Biol. Chem. 53: 204-213, 2014.

Yang, L., Xu, M., Koo, Y., He, J., Poethig, R.S.: Sugar promotes vegetative phase change in Arabidopsis thaliana by repressing the expression of MIR156A and MIR156C. - eLife 2: e00260, 2013. 